\title{
Somatic copy number alterations have prognostic impact in patients with ovarian clear cell carcinoma
}

\author{
ASUKA MORIKAWA ${ }^{1,2}$, TOMOATSU HAYASHI $^{1}$, MANA KOBAYASHI $^{1}$, YUKI KATO $^{3}$, \\ KATSUHIKO SHIRAHIGE ${ }^{3}$, TAKEHIKO ITOH ${ }^{4}$, MITSUYOSHI URASHIMA ${ }^{5}$, \\ AIKOU OKAMOTO $^{2}$ and TETSU AKIYAMA ${ }^{1}$
}

\begin{abstract}
${ }^{1}$ Laboratory of Molecular and Genetic Information, Institute of Molecular and Cellular Biosciences, The University of Tokyo, Tokyo 113-0032; ${ }^{2}$ Department of Obstetrics and Gynecology,

Jikei University School of Medicine, Tokyo 105-8461; ${ }^{3}$ Laboratory of Genome Structure and Function,

Research Center for Epigenetic Disease, Institute for Molecular and Cellular Biosciences, The University of Tokyo, Tokyo 113-0032; ${ }^{4}$ Graduate School of Bioscience and Biotechnology, Tokyo Institute of Technology, Tokyo 152-8550;

${ }^{5}$ Division of Molecular Epidemiology, Jikei University School of Medicine, Tokyo 105-8461, Japan
\end{abstract}

Received November 17, 2017; Accepted April 17, 2018

DOI: $10.3892 /$ or.2018.6419

\begin{abstract}
Ovarian clear cell carcinoma (OCCC) is a chemotherapy-resistant epithelial ovarian cancer with poor prognosis. To identify genomic alterations involved in the development of OCCC, we analyzed somatic copy number alterations in OCCC using comparative genomic hybridization $(\mathrm{CGH})$. Here we showed that the chromosomal regions 8p11.21, 8p11.22, 12p13.31 and 20q13.2 were amplified in OCCC. We also demonstrated that small segments in the chromosomal regions 3q26.1, 4q13.2 and 22q11.23 were deleted. Kaplan-Meier survival analyses revealed that patients with amplification within 8p11.21 or a deletion within 3q26.1 had a shorter progression-free survival (PFS) time than those without such alterations. In addition, patients with amplification in three of the four chromosomal regions $8 \mathrm{p} 11.21,8 \mathrm{p} 11.22$, $12 \mathrm{p} 13.31$ and 20q13.2 had shorter overall survival (OS). We also demonstrated that amplification of $12 \mathrm{p} 13.3$ or three of the four chromosomal regions 8p11.21, 8p11.22, 12p13.31 and $20 \mathrm{q} 13.2$, or a deletion in the chromosomal region $3 \mathrm{q} 26.1$ was associated with chemotherapy resistance. Our findings suggest that copy number alterations in $8 \mathrm{p} 11.21-22,12 \mathrm{p} 13.31,20 \mathrm{q} 13.2$, $3 q 26.1,4 q 13.2$ and $22 \mathrm{q} 11.23$ are critical for the development and survival of OCCC.
\end{abstract}

Correspondence to: Dr Tetsu Akiyama, Laboratory of Molecular and Genetic Information, Institute of Molecular and Cellular Biosciences, The University of Tokyo, 1-1-1 Yayoi, Bunkyo-ku, Tokyo 113-0032, Japan

E-mail: akiyama@iam.u-tokyo.ac.jp

Key words: ovarian cancer, ovarian clear cell carcinoma, amplification, somatic copy number, prognosis, comparative genomic hybridization

\section{Introduction}

Epithelial ovarian cancer is classified into four major subgroups: serous, clear cell, endometrioid and mucinous (1). Ovarian clear cell carcinoma (OCCC) has phenotypes distinct from those of the other subgroups, such as resistance to chemotherapy, poor prognosis, an association with endometriosis, a higher incidence of thrombosis as a complication and a higher incidence among Japanese individuals (2-4). OCCC contains mutations in various tumor suppressors and oncogenes, including AT-rich interactive domain-containing protein 1A (ARID1A), phosphatidylinositol-3-kinase catalytic subunit (PIK3CA), KRAS and TP53 (5). Furthermore, a gain in DNA copy number is frequently found in chromosome 20, which contains the potential oncogene zinc finger protein 217 (ZNF217), while losses in DNA copy number are observed at the CDKN2A/2B and LZTS1 loci $(6,7)$.

We recently analyzed somatic copy number alterations in OCCC using comparative genomic hybridization (CGH) (8). In agreement with previous reports (7), we found recurrent amplification of the 20q13 locus containing ZNF217 and the 17q23.2 locus harboring PPM1D. Furthermore, we found recurrent amplification of the entire chromosome $8 \mathrm{q}$, the EGFR and HER2/ERBB2 gene loci, and losses of chromosomes $9 \mathrm{q}, 13 \mathrm{q}$ and $17 \mathrm{q}$. In the present study, we reanalyzed this CGH data and further identified amplification in the chromosomal regions $8 \mathrm{p} 11.21-22$ and $12 \mathrm{p} 13.31$ as well as 20q13.2, and losses of small segments in the chromosomal regions 3q26.1, 4q13.2 and 22q11.23 in OCCC. Moreover, we showed that these DNA copy number alterations are associated with poor patient prognosis.

\section{Materials and methods}

Specimens. Tumor specimens were surgically obtained from 110 OCCC patients (95 Japanese and 15 Korean patients). All the patients provided written informed consent. This study was 
Table I. Characteristics of the OCCC patients.

\begin{tabular}{|c|c|}
\hline Characteristics & Data \\
\hline Total patients, $\mathrm{n}$ & 110 \\
\hline Age (years), median (range) & $53(30-86)$ \\
\hline \multicolumn{2}{|l|}{ Stage, $\mathrm{n}$} \\
\hline $\mathrm{I}$ & 61 \\
\hline II & 14 \\
\hline III & 32 \\
\hline IV & 3 \\
\hline Endometriosis, n (\%) & $54(49)$ \\
\hline Thrombosis, n (\%) & $12(11)$ \\
\hline \multicolumn{2}{|l|}{ Sensitivity to chemotherapy, n (\%) } \\
\hline Sensitive & $60(55)$ \\
\hline Resistant & $26(24)$ \\
\hline Disease duration (months), median (IQR) & $17.8(10-36)$ \\
\hline Progression, n (\%) & $33(30)$ \\
\hline Deceased, n (\%) & $21(19)$ \\
\hline \multicolumn{2}{|l|}{ Amplification, n (\%) } \\
\hline $8 p 11.22$ & $20(18)$ \\
\hline $8 p 11.21$ & $14(13)$ \\
\hline $12 \mathrm{p} 13.31$ & $13(12)$ \\
\hline $20 \mathrm{q} 13.2$ & $14(13)$ \\
\hline \multicolumn{2}{|l|}{ Deletion } \\
\hline $3 q 26.1$ & $36(33)$ \\
\hline $4 q 13.2$ & $21(19)$ \\
\hline $22 q 11.23$ & $4(4)$ \\
\hline
\end{tabular}

OCCC, ovarian clear cell carcinoma.

performed in accordance with the Helsinki Declaration and was approved by the Ethics Committee of the Jikei University School of Medicine (8). The characteristics of the patients are summarized in Table I. Our study sample was considered to be representative of the OCCC patient population. Japanese and Korean individuals are genetically very similar (9). The response to chemotherapy was defined as: i) platinum sensitive, if no relapse or progression was noted within 6 months after the last cycle of chemotherapy; ii) platinum resistant, if the patients did not respond to therapy or responded initially but relapsed or progressed within 6 months after the last cycle of chemotherapy.

CGH analysis. CGH analysis was performed according to the manufacturer's instructions (Agilent Technologies, Inc., Santa Clara, CA, USA). DNA was extracted from tumor and normal tissues using the AllPrep DNA/RNA Mini kit and the QIAamp DNA Mini kit, respectively (Qiagen, Dusseldorf, Germany). Tumor and normal DNA digested with Rsa1 and Alu1 was labeled with $\mathrm{Cy} 5$ and $\mathrm{Cy} 3$, respectively, using the Agilent Genomic DNA Enzymatic Labeling kit (Agilent Technologies, Inc.). The labeled DNA was hybridized to Human Genome CGH Microarray kit 244A (G4411B; Agilent Technologies, Inc.), which contains $\sim 236,381$ probes
Table II. Primer/probe sequences.

\begin{tabular}{ll}
\hline Gene & \multicolumn{1}{c}{ Sequences } \\
\hline ANK1 & \\
Forward & 5'-TCCCCTGAATTAAGCCTTCAG-3' \\
Reverse & 5'-CCACCCCAGGGACTCTTTAC-3' \\
Probe & ROCHE UPL \#55 \\
MYST3 & \\
Forward & 5'-CATGTTGTTTTCCCCTTTCAA-3' \\
Reverse & 5'-ACGAAAAAGAAATTCCAACTGTG-3' \\
Probe & ROCHE UPL \#69 \\
IKBKB & \\
Forward & 5'-ATCATTGTGGGCTGCAGATT-3' \\
Reverse & 5'-TGGGGAATCCTCTCTCCACT-3' \\
Probe & ROCHE UPL \#19 \\
POLB & \\
Forward & 5'-TTGAACCATCATCAGCGAAT-3' \\
Reverse & 5'-CTCAAGTGTCAAAAGAAAATCTGC-3' \\
Probe & ROCHE UPL \#41 \\
ZNF217 & \\
Forward & 5'-CACGATTGATTGGACTCTTCC-3' \\
Reverse & 5'-CACGTCAATCACATGATCAGAA-3' \\
Probe & ROCHE UPL \#41 \\
ADAM5 & \\
Forward & 5'-CCAGTGCCTAGAAGAGTGTCTG-3' \\
Reverse & 5'-CAGACTCTGTGACTCCTTTGTATCA-3' \\
Probe & ROCHE UPL \#5 \\
Chr12 & \\
ENST00000518709 \\
Forward & 5'-CTGTAGCCATCTGTCCAAGTGT-3' \\
Reverse & 5'-AGGCAAGCAGAGGAAATCTG-3' \\
Probe & ROCHE UPL \#19 \\
& \\
\hline
\end{tabular}

ZNF217, zinc finger protein 217.

annotated against the National Center for Biotechnology Information (NCBI) Build 36. The array was scanned with an Agilent G2565BA Microarray Scanner, and the fluorescent signals were analyzed using Feature Extraction software, version 10.7.3.1 (Agilent Technologies, Inc.). DNA copy number aberrations were identified using the ADM-2 algorithm in CytoGenomics Software (Agilent Technologies, Inc.). Regions with a copy number gain were defined as those with an average $\log _{2}$ ratio $\geq 0.9$ across at least three consecutive probes. Regions with a copy number loss were defined as those with an average $\log _{2}$ ratio $\leq-1.5$ across at least three consecutive probes. All genomic positions were defined according to the University of California Santa Cruz (UCSC) Human version hg19. The P-value corresponding to each interval was calculated using the normal probability distribution function and the score of that interval. The abnormal chromosomal regions among the OCCC population were defined by applying T-Test Common Aberration algorithm (Genomic Workbench; Agilent Technologies, Inc.). 

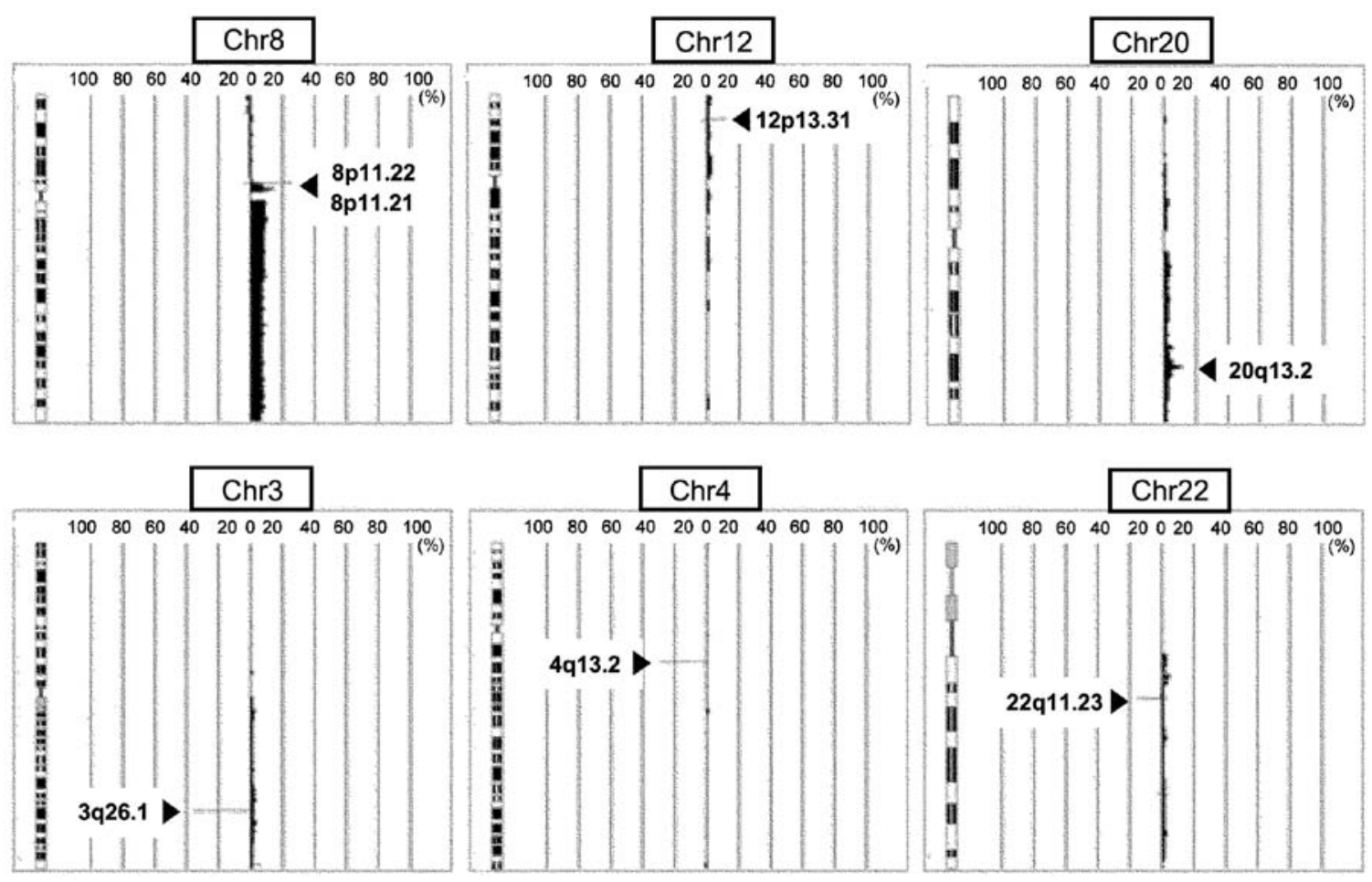

Figure 1. Identification of copy number aberrations in 110 OCCC patients by CGH analysis. Frequencies (\%) of copy number gains (right side of the central axis) and losses (left side of the central axis) are shown. Dotted lines on the right side of each chromosome ideogram indicate the frequency (0-100\%) of the identified aberrations. OCCC, ovarian clear cell carcinoma; CGH, comparative genomic hybridization.

Droplet digital PCR (ddPCR). DNA was extracted from tumor tissues and buffy coat samples using the AllPrep DNA/RNA Mini kit and the QIAamp DNA Mini kit, respectively (Qiagen). Probes were from the Universal Probe Library (UPL) (Roche Diagnostics, Basel, Switzerland). A minor groove-binding (MGB) probe labeled with 6-carboxyfluorescein (FAM) was used for ddPCR to assess the copy number of ANK1, MYST3, IKBKB, POLB and ZNF217. A MGB probe labeled with VIC (Thermo Fisher Scientific, Inc., Waltham, MA, USA) was used to quantitate endogenous control EFTUD2. Primers of $\sim 20$ bases were designed using Primer3Plus and purchased from Invitrogen (Thermo Fisher Scientific, Inc.). Primer sequences are listed in Table II. Reaction mixtures $(20 \mu \mathrm{l})$ containing 5 or $10 \mathrm{ng}$ of digested sample DNA, ddPCR supermix for probes (Bio-Rad Laboratories, Inc., Hercules, CA, USA), 1,000 nM of each primer and $250 \mathrm{nM}$ of each probe were loaded into the QX100 Droplet Generator (10). The samples were amplified on the conventional Bio-Rad T100 Thermal Cycler $\left(95^{\circ} \mathrm{C}\right.$ for $10 \mathrm{~min}$, followed by 40 cycles of $95^{\circ} \mathrm{C}$ for $30 \mathrm{sec}$ and $60^{\circ} \mathrm{C}$ for $60 \mathrm{sec}$, with a final elongation step of $98^{\circ} \mathrm{C}$ for $10 \mathrm{~min}$ ). The plate, containing the droplet amplicons, was subsequently loaded into the QX100 Droplet Reader (Bio-Rad Laboratories, Inc.). The number of gene copies was calculated using QuantaSoft software (Bio-Rad Laboratories, Inc.). The average number of droplets generated was 12,136 . Data were analyzed using the Wilcoxon rank sum test.

Exome capture library and whole-exome sequencing. DNA libraries were generated from genomic DNA derived from tumor and/or normal tissues of the OCCC patients, and subsequently sequenced. From this, exome-captured sequencing libraries were produced using Agilent SureSelect XT Human All Exon v5 (Agilent Technologies, Inc.). The captured DNA was sequenced using the Illumina HiSeq 2500 platform (Illumina, Inc., San Diego, CA, USA) with paired-end reads of $100 \mathrm{bp}$ for insert libraries according to the manufacturer's instructions. We deposited all DNA sequence data used in this study to the National Bioscience Database Center (NBDC) Human Database (http://humandbs.biosciencedbc.jp/).

Exome sequence data analysis. Low quality $(\mathrm{q}<15)$ and adaptor regions were trimmed using in-house software beforehand. Read sequences were mapped using Bowtie 2 (11) to the human reference genome (B37). After filtering by pair mapping distance, mapping uniqueness and pair orientation, the mapping result files were converted into pileup format using SAMtools (12). Copy number changes, or amplifications and deletions were detected from coverage comparisons between tumor and normal data sets. Focal gains and losses were defined as occurring on gene segments.

Kaplan Meier analysis. Progression-free survival (PFS) rates and overall survival (OS) rates were calculated using the Kaplan-Meier method and evaluated using the log-rank test. Confidence intervals (CIs) were assessed by univariate and multivariate Cox proportional hazards models. Multivariate Cox models were used to determine whether the increased hazard rates for PFS and OS attributed to gene amplification in the univariate model were still present after accounting for patient-, disease-, and prior treatment-related factors. $\mathrm{P}<0.05$ was considered to be statistically significant. 


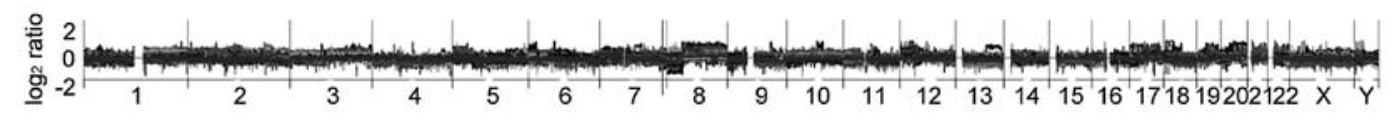

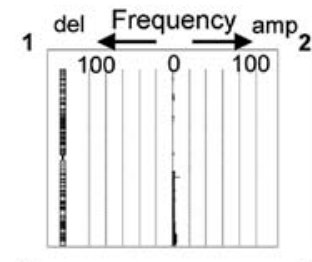

6
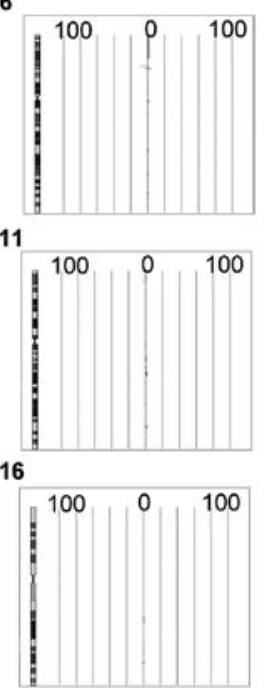

21

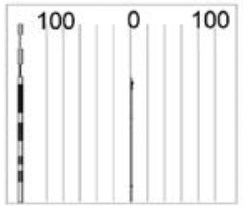

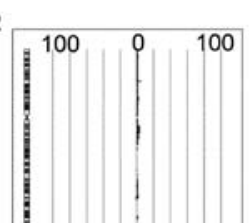

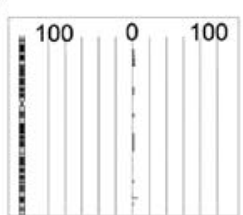

12
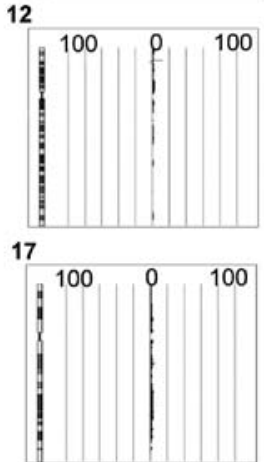

22

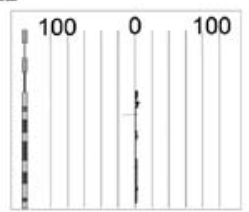

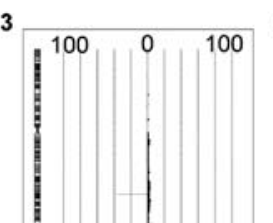

8

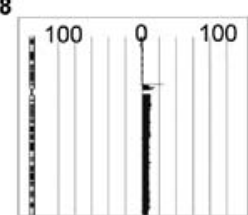

13

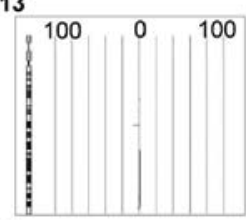

18

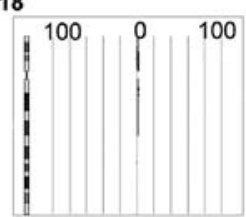

$\mathbf{x}$

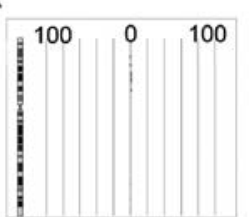

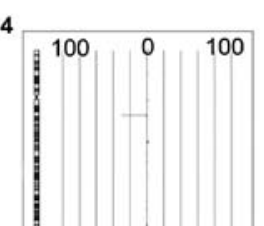

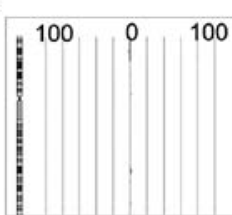

14
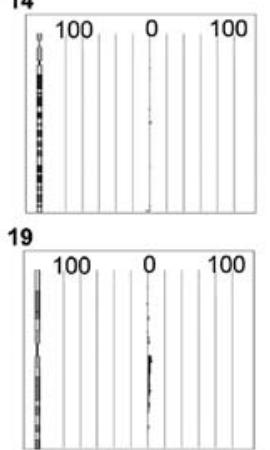

$\mathbf{Y}$

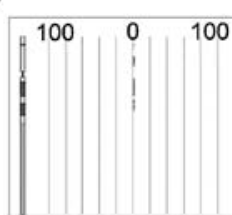

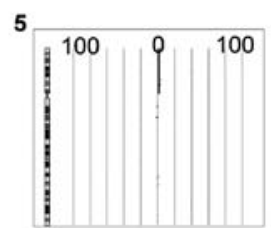

10

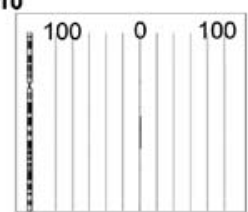

15

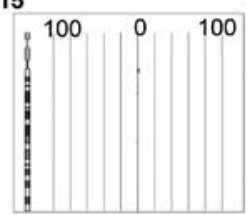

20

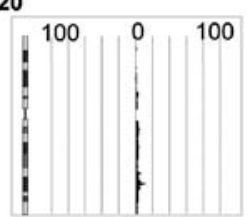

Figure 2. Identification of copy number aberrations in 110 OCCC patients by CGH analysis (all chromosome). OCCC, ovarian clear cell carcinoma; CGH, comparative genomic hybridization.

\section{Results}

The characteristics of the 110 patients (95 Japanese and 15 Korean patients) studied are summarized in Table I. With a median follow-up of 17.8 months (range, 10-36 months), 33 (30\%) patients relapsed and $21(19 \%)$ patients died. The mean age was 53 years (range, 30-86 years). FIGO stages were: stage I, 61 patients; stage II, 14 patients; stage III, 32 patients; stage IV, 3 patients. Endometriosis was diagnosed in 54 (49\%) patients. Thrombosis was observed in $12(11 \%)$ patients. The response to chemotherapy was: platinum sensitive, $60(55 \%)$ patients; platinum resistant, 26 (24\%) patients.

In our previous CGH analysis of somatic copy number alterations in OCCC (8), we selected regions with a minimum average $\log _{2}$ ratio \pm 0.25 (8). In the present study, we reanalyzed the CGH data from 110 OCCC patients: regions with an average $\log _{2}$ ratio $\geq 0.9$ or $\leq-1.5$ were defined as those with a copy number gain or loss, respectively (Figs. 1 and 2). We identified increases in DNA copy number of the chromosomal region 8p11.22, which harbors ADAM5p and ADAM3A, in tumor tissues from $\sim 25 \%$ of patients; 8p11.21, harboring a number of genes, including ANK1, MYST3/KAT6A, IKBKB, POLB and DKK4, in $14-16 \%$ of patients; 12p13.31, harboring the long non-coding RNAs (lncRNAs) ENST00000518709 and ENST00000545710, in $\sim 13 \%$ of patients; and 20q13.2, harboring the candidate oncogene ZNF217, in $15 \%$ of patients (Fig. 1 and Table III). In addition, we identified losses of small segments (regions with an average signal ratio $\leq-0.5$ ) in the chromosomal regions $8 \mathrm{p} 11.22$ and 12p13.31 in normal samples from 5 and $17 \%$ of OCCC patients, respectively.

We also identified losses of small segments in the chromosomal region $3 \mathrm{q} 26.1$, which harbors the lncRNA BC073807; 4q13.2, harboring UDP-glucuronosyltransferase 2B17 (UGT2B17); and 22q11.23, harboring glutathione S-transferase $\theta 1$ (GSTT1) and D-dopachrome tautomerase-like (LOC391322), in tumor tissues from 33, 19 and 4\% patients, respectively (Fig. 1 and Table III). In addition, we identified losses of small segments in the chromosomal regions $3 \mathrm{q} 26.1$, $4 q 13.2$ and 22q11.23 in normal samples from 45, 36 and $24 \%$ patients, respectively.

To validate these results, we performed ddPCR analysis of copy number for ANK1, MYST3, IKBKB and POLB in four OCCC patients (Fig. 4, left panels). Consistent with the results of the $\mathrm{CGH}$ analysis, these genes were amplified in three OCCC patients with 8p11.21 amplification, OC19, OC69 and OC96, but not in a patient without 8p11.21 
Table III. Copy number aberrations detected by CGH analysis in 110 OCCC patient tissues.

\begin{tabular}{|c|c|c|c|c|c|c|}
\hline \multirow[b]{2}{*}{ Chr no. } & \multirow[b]{2}{*}{ Cytoband } & \multicolumn{2}{|c|}{ Region } & \multirow[b]{2}{*}{ Genes } & \multirow{2}{*}{$\begin{array}{c}\text { No. of } \\
\text { patients, } \mathrm{n}(\%)\end{array}$} & \multirow[b]{2}{*}{ P-value } \\
\hline & & Start & End & & & \\
\hline \multicolumn{7}{|c|}{$\begin{array}{l}\text { Amplification } \\
\log _{2} \geq 0.9\end{array}$} \\
\hline \multirow[t]{3}{*}{ Chr8 } & 8p11.22 & 39237438 & 39386158 & ADAM5p/ADAM3A & $28(25)$ & $1.928 \mathrm{E}-25$ \\
\hline & $8 \mathrm{p} 11.21$ & 41640598 & 41889042 & ANK1/MYST3 & $16(15)$ & $1.647 \mathrm{E}-17$ \\
\hline & & 42033118 & 42543909 & $\begin{array}{l}\text { PLAT/IKBKB/POLB/DKK4 } \\
\text { /VDAC/SLC20A2/SMIM19 }\end{array}$ & $14(13)$ & \\
\hline Chr12 & $12 \mathrm{p} 13.31$ & 9637323 & 9693948 & $\begin{array}{l}\text { ENST00000518709 } \\
/ \text { ENST00000545710 }\end{array}$ & $13(12)$ & $5.38 \mathrm{E}-19$ \\
\hline Chr20 & $20 \mathrm{q} 13.2$ & 52163628 & 52215265 & ZNF217 & $14(13)$ & $6.349 \mathrm{E}-26$ \\
\hline \multicolumn{7}{|l|}{$\begin{array}{l}\text { Deletion } \\
\log _{2} \leq 1.5\end{array}$} \\
\hline Chr3 & $3 q 26.1$ & 162514534 & 162619141 & ВС073807 & $36(33)$ & $2.253 \mathrm{E}-34$ \\
\hline Chr4 & $4 q 13.2$ & 69375140 & 69483277 & UGT2B17 & $21(19)$ & $5.293 \mathrm{E}-27$ \\
\hline Chr22 & $22 q 11.23$ & 24371205 & 24390254 & GSTT1/LOC391322 & $4(4)$ & $3.94 \mathrm{E}-15$ \\
\hline
\end{tabular}

CGH, comparative genomic hybridization; OCCC, ovarian clear cell carcinoma; ZNF217, zinc finger protein 217; UGT2B17, UDP-glucuronosyltransferase 2B17; GSTT1, glutathione S-transferase $\theta$ 1; LOC391322, D-dopachrome tautomerase-like.
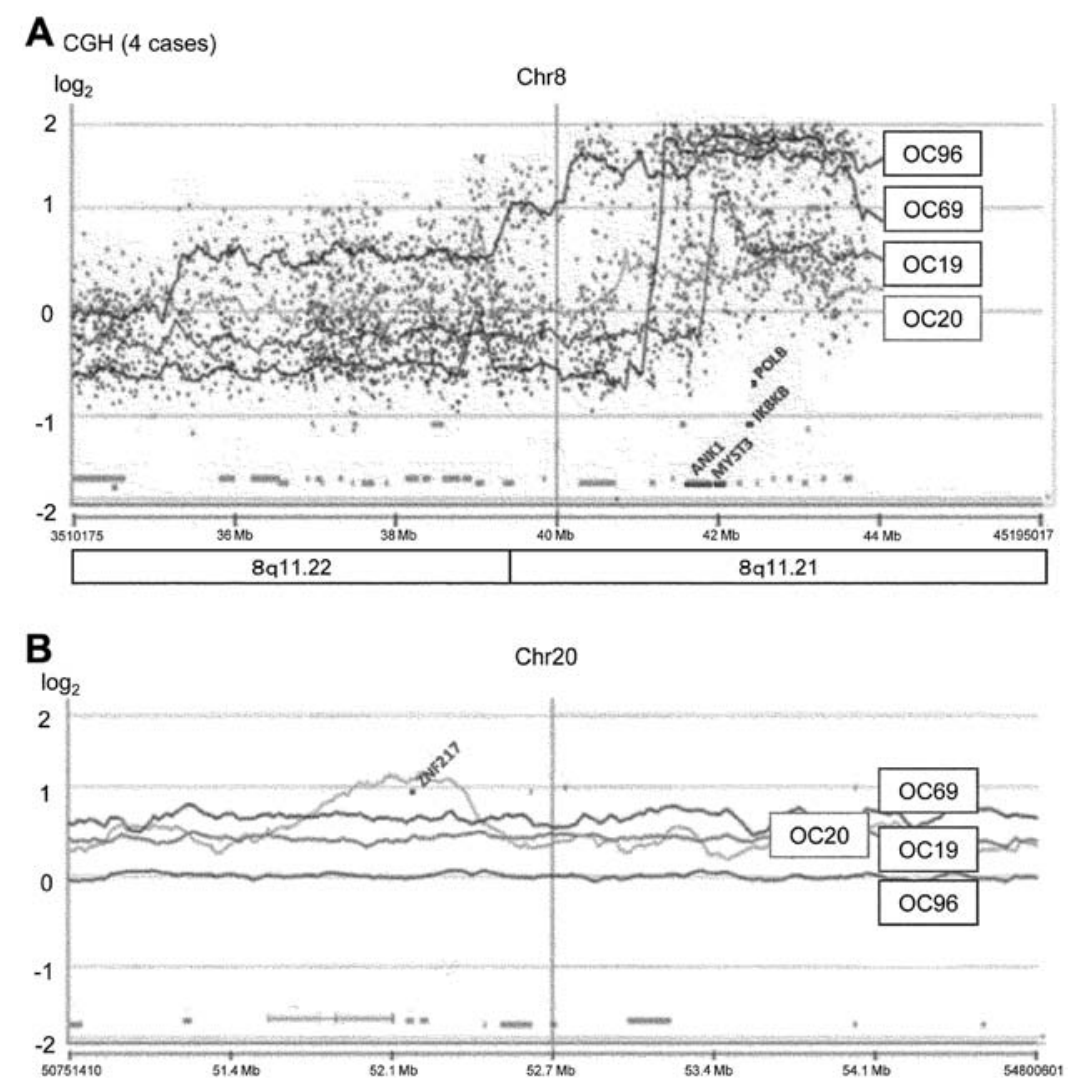

Figure 3. CGH profile of chromosome (A) 8 and (B) 20 showing amplification in 4 OCCC patients. CGH, comparative genomic hybridization; OCCC, ovarian clear cell carcinoma.

amplification, OC20 (Fig. 3). We also confirmed that ZNF217 was amplified in a patient with 20q13.2 amplification, OC20. Analysis of 9 patients confirmed the amplification of these genes (Fig. 4; 4 patients in the left panels and 5 patients in the right panels). ADAM5p and the lncRNA ENST00000518709 appeared to be amplified, but no statistical significance was 

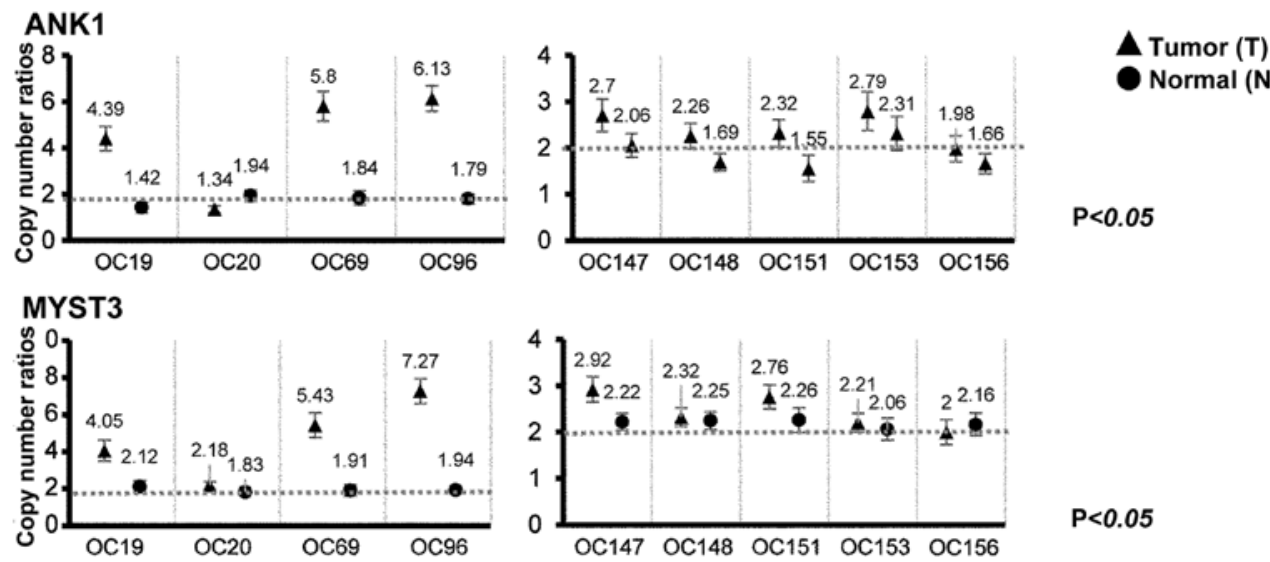

$\mathrm{P}<0.05$

\section{IKBKB}

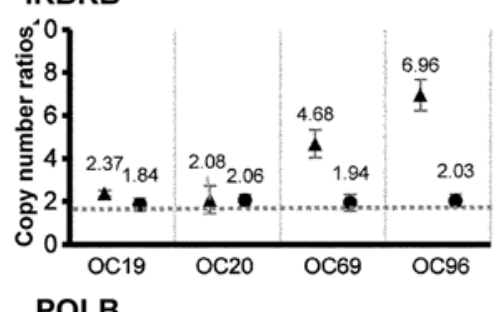

POLB
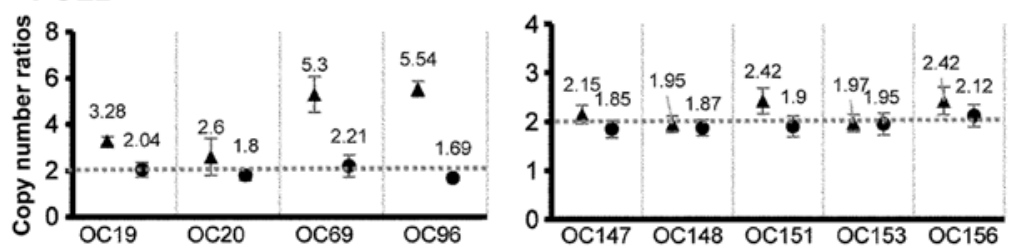

$\mathrm{P}<0.05$

$\mathrm{P}<0.01$
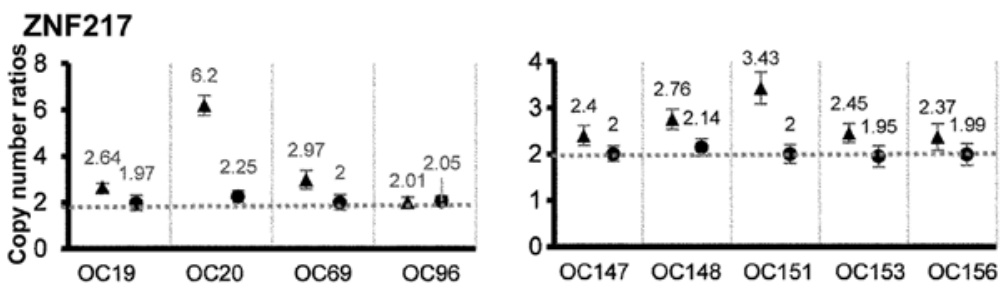

$P<0.01$

ADAM5

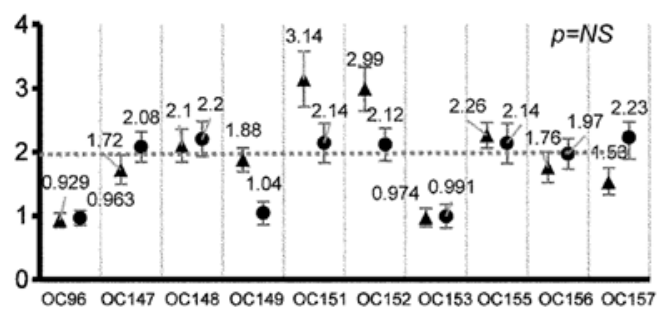

Chr12 non-coding RNA (ENST00000518709)

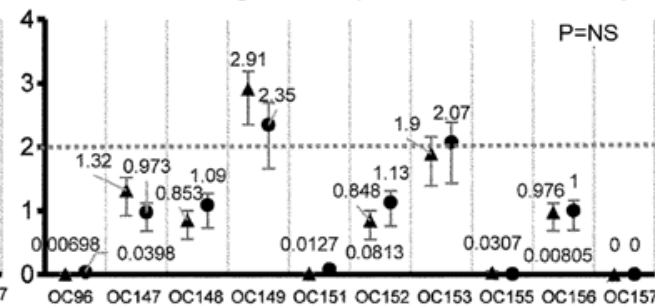

Figure 4. ddPCR analysis of copy number aberrations in OCCC patients. Copy number of the indicated genes was measured by ddPCR. Among patients analyzed in this figure, OC19, OC20, OC69 and OC96 were patients analyzed by CGH in Table IV and Fig. 3. EFTUD2 was used as a reference. Error bars represent 95\% CI. P-values are based on the Wilcoxon rank sum test. ddPCR, droplet digital PCR; OCCC, ovarian clear cell carcinoma; CGH, comparative genomic hybridization; CI, confidence interval.

found (Fig. 4). In addition, consistent with the results of CGH analysis, we observed losses of ADAM5p and the lncRNA ENST00000518709 in normal samples in 3 out of 10 and 7 out of 9 OCCC patients, respectively. Furthermore, we performed exome sequence analysis of 3 patients and confirmed that 8p11.21, but not 20q13.2, was amplified in OC96 (Fig. 5 and Table IV).

We finally investigated the relationship between the amplification or loss of these genes and survival, sensitivity to
chemotherapy,thrombosisandendometriosis.TheKaplan-Meier survival analysis showed that patients with amplification of chromosome $8 \mathrm{p} 11.21$ had shorter PFS than those without such amplification ( $\mathrm{P}=0.057,95 \% \mathrm{CI}$ : 0.961-5.154) (Fig. 6A). Patients with amplification in three of the four chromosomal regions 8p11.21, 8p11.22, 12p13.31 and 20q13.2 had shorter OS ( $\mathrm{P}=0.068,95 \%$ CI: $0.833-16.393)$ as well as shorter PFS $(\mathrm{P}=0.001,95 \% \mathrm{CI}$ : 1.615-13.698) (Fig. 6B and C, Table V). Patients with a deletion in the chromosomal region $3 \mathrm{q} 26.1$ 

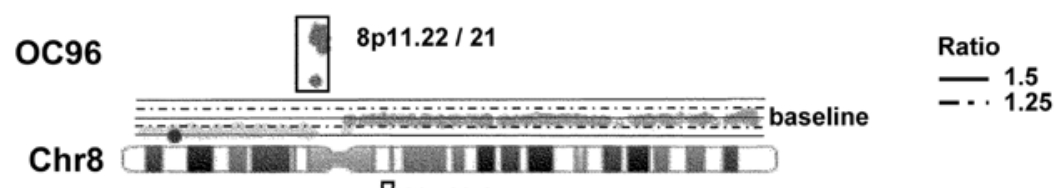

Chr8

\section{Chr20}
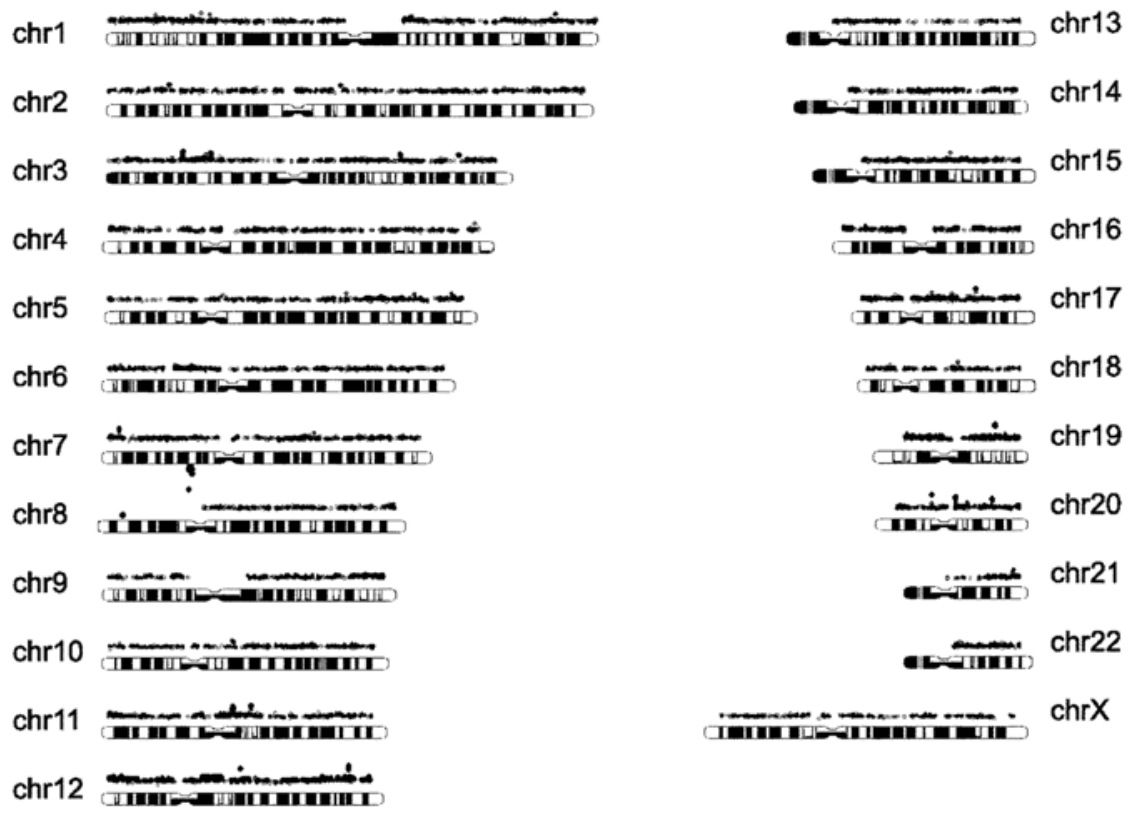

Figure 5. Exome sequence analysis of copy number aberrations in patient OC96. Frequencies (\%) of copy number gains (above the center line) and losses (below the center line) are shown.

had shorter PFS (P=0.046, 95\% CI: 0.998-4.000) (Fig. 6D). Analysis of the correlation between copy number alterations and sensitivity to chemotherapy revealed that amplification of $12 \mathrm{p} 13.3$ or three of the four chromosomal regions $8 \mathrm{p} 11.21$, 8 p11.22, 12p13.31 and 20q13.2 was associated with resistance to platinum-based chemotherapy $(\mathrm{P}=0.011$ or 0.012 , respectively) (Table VI). We also found that a deletion in the chromosomal region 3q26.1 was associated with chemotherapy resistance $(\mathrm{P}=0.008)$ (Table $\mathrm{VI})$. We did not observe any association between copy number alterations and sensitivity to chemotherapy, FIGO stage, thrombosis or endometriosis.

\section{Discussion}

In the present study, we showed that the chromosomal regions 8p11.21, 8p11.22 and 12p13.31 are amplified and the chromosomal regions 3q26.1, 4q13.2 and 22q11.23 are deleted in OCCC. Kaplan-Meier survival analyses revealed that patients with amplification within 8 p11.21 or a deletion within $3 \mathrm{q} 26.1$ had a shorter PFS than those without such copy number alterations. Furthermore, patients with amplification in three of the four chromosomal regions 8p11.21, 8p11.22, 12p13.31 and $20 \mathrm{q} 13.2$ had shorter OS. Consistent with these results, we found that amplification of $12 \mathrm{p} 13.3$ or three of the four chromosomal regions $8 \mathrm{p} 11.21,8 \mathrm{p} 11.22,12 \mathrm{p} 13.31$ and $20 \mathrm{q} 13.2$, or a deletion in the chromosomal region $3 \mathrm{q} 26.1$ is associated with chemotherapy resistance (Table VI). Thus, amplification in 8p11.21, 8p11.22, 12p13.31 and 20q13.2 and a deletion in the chromosomal region 3 q26.1 may be critical for the survival of OCCC patients.

The chromosomal region 8p11.21 encodes a number of genes, including ANK1, MYST3, IKBKB and POLB, which have been reported to play important roles in the development of a number of cancers $(13,14)$. It is therefore possible that amplification of the genes encoded in this region contribute to the development of OCCC.

ADAM5p and ADAM3A encoded in the chromosomal region 8p11.2 are pseudogenes of the ADAM metallopeptidases (15). It has recently been reported that pseudogenes can function as lncRNAs, which can play important roles in tumorigenesis. For example, PTENP1 functions as a competing endogenous RNA to suppress tumor progression (16). UPAT, which is encoded by the pseudogene of the amine oxidase copper containing-3 (AOC3) gene, interacts with and stabilizes the epigenetic factor UHRF1 by interfering with its $\beta$-TrCP-mediated ubiquitination, thereby promoting the tumorigenicity of colorectal cancer cells (17). In addition, it has been reported that the chromosomal region 8p11.22 is amplified and ADAM5p and ADAM3A are overexpressed in conjunctival squamous cell carcinoma and glioma $(18,19)$. We also found that the lncRNA ENST00000518709 is amplified in OCCC patients. Thus, it is possible that amplification of these lncRNAs is important for the development and progression of OCCC. In addition, we observed losses of 8p11.22 and/or 12 p13.31 in normal samples from 5 and $17 \%$ of OCCC patients, 
Table IV. Analyses of copy number aberrations by CGH array, ddPCR and Exome-seq.

\begin{tabular}{|c|c|c|c|c|c|c|}
\hline \multirow{2}{*}{$\begin{array}{l}\text { Gene/ } \\
\text { method }\end{array}$} & \multicolumn{6}{|c|}{ Tumor/normal ratio } \\
\hline & OC19 & OC20 & OC69 & OC96 & OC147 & OC148 \\
\hline \multicolumn{7}{|l|}{ ANK1 } \\
\hline CGH array & 1.51 & 1.22 & 2.43 & 3.20 & & \\
\hline ddPCR & 3.09 & 0.69 & 3.15 & 3.42 & 1.31 & 1.33 \\
\hline Exome-seq & & & & 3.66 & 0.83 & 1.20 \\
\hline \multicolumn{7}{|l|}{ MYST3 } \\
\hline CGH array & 2.14 & 1.22 & 2.42 & 3.20 & & \\
\hline ddPCR & 1.91 & 1.19 & 2.84 & 3.75 & 1.31 & 1.01 \\
\hline Exome-seq & & & & 3.5 & 1.22 & 1.18 \\
\hline \multicolumn{7}{|l|}{ IKBKB } \\
\hline CGH array & 1.40 & 1.22 & 2.17 & 3.20 & & \\
\hline ddPCR & 1.28 & 1.00 & 2.41 & 3.74 & & \\
\hline Exome-seq & & & & 3.55 & & \\
\hline \multicolumn{7}{|l|}{ POLB } \\
\hline CGH array & 1.41 & 1.22 & 2.18 & 3.20 & & \\
\hline ddPCR & 1.60 & 1.44 & 2.39 & 3.28 & 1.16 & 1.04 \\
\hline Exome-seq & & & & 3.54 & 1.19 & 1.19 \\
\hline \multicolumn{7}{|l|}{ ZNF217 } \\
\hline CGH array & 1.26 & 2.02 & 1.27 & 1.03 & & \\
\hline ddPCR & 1.34 & 2.75 & 1.45 & 0.98 & 1.2 & 1.31 \\
\hline Exome-seq & & & & 0.97 & 1.19 & 1.36 \\
\hline
\end{tabular}

CGH, comparative genomic hybridization; ddPCR, droplet digital PCR; Exome-seq, exome sequencing; ZNF217, zinc finger protein 217.

respectively. The significance of these losses in normal tissues remains to be investigated.

While we also found that the chromosomal regions $3 \mathrm{q} 26.1,4 \mathrm{q} 13.2$ and 22q11.23 were deleted in 30, 19 and $4 \%$ of OCCC, respectively, we also identified losses of small segments in these chromosomal regions in normal samples from 45,36 and $24 \%$ of patients, respectively. CGH analysis did not discriminate between the segments deleted in normal samples and those deleted only in tumor tissues. These loci encode the lncRNA BC073807, UGT2B17 (a member of the uridine diphosphoglucuronosyltransferase protein family), and GSTT1 and LOC391322, respectively. It remains to be investigated whether deletion of these genes contributes to the development of OCCC. It is also possible that deletion of these regions may disrupt the three-dimensional networks of chromosomal interactions and thereby lead to altered gene expression (20-22). Elucidation of the functional significance of these deletions in the development of OCCC is underway in our laboratories.

In conclusion, we identified somatic copy number alterations that have prognostic value in patients with OCCC. We also identified copy number alterations associated with chemotherapy resistance. However, it remains to be confirmed whether amplification of these genes indeed results in changes in the expression of encoded proteins. Furthermore, it remains
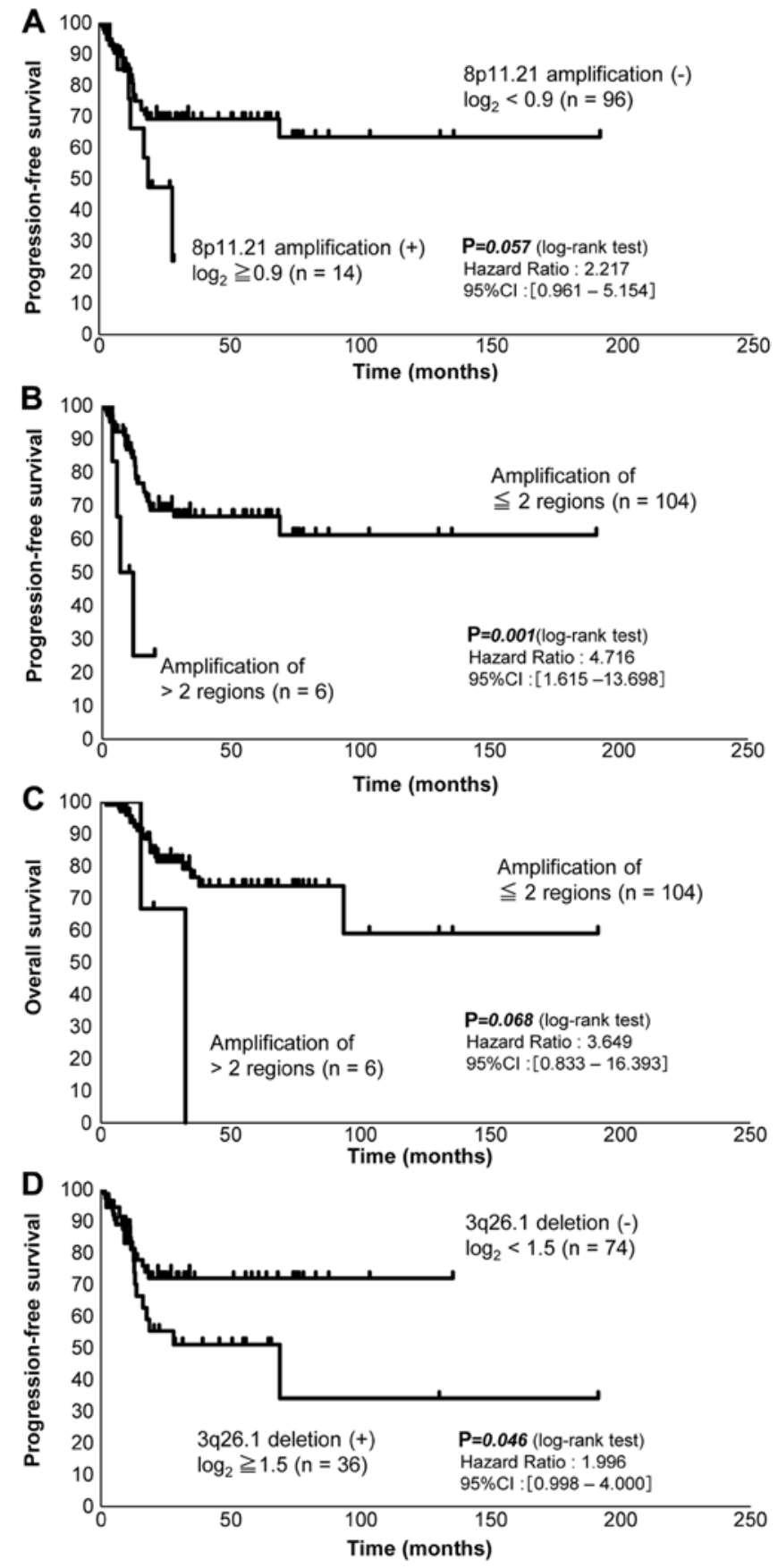

Figure 6. Kaplan-Meier analysis of (A, B and D) PFS and (C) OS of OCCC patients. (A) Patients with 8p11.21 amplification. (B and C) Patients with amplification in three of the four chromosomal regions 8p11.21, 8p11.22, 12p13.31 and 20q11.23. (D) Patients with 3q26.1 deletion. PFS, progression-free survival; OS, overall survival; OCCC, ovarian clear cell carcinoma.

to be seen whether amplification or deletion of these genes contributes to the development, progression and/or chemosensitivity of OCCC. The identification and characterization of these genes could provide novel insights into the mechanisms of OCCC development and chemosensitivity. We speculate that these genes could be promising molecular targets and/or markers for OCCC therapy.

\section{Acknowledgements}

Not applicable. 
Table V. Multivariate analysis of prognostic factors for 110 OCCC patients $(n=110)$.

\begin{tabular}{|c|c|c|c|c|c|c|c|}
\hline \multirow[b]{2}{*}{ Factors } & \multirow[b]{2}{*}{ Category } & \multicolumn{3}{|c|}{ Progression-free survival } & \multicolumn{3}{|c|}{ Overall survival } \\
\hline & & $\mathrm{HR}$ & $95 \% \mathrm{CI}$ & P-value & $\mathrm{HR}$ & $95 \% \mathrm{CI}$ & $\mathrm{P}$-value \\
\hline Age (years) & $<60 / \geq 60$ & 1.125 & $0.462-2.740$ & 0.795 & 0.797 & $0.288-2.212$ & 0.664 \\
\hline FIGO stage & III-IV/I-II & 6.211 & $2.949-12.987$ & $<0.001$ & 3.937 & $1.612-9.615$ & 0.003 \\
\hline Race & Japanese/Korean & 1.204 & $0.408-3.558$ & 0.735 & 1.128 & $0.245-5.181$ & 0.644 \\
\hline Endometriosis & Absence/presence & 1.949 & $0.906-4.195$ & 0.088 & 2.122 & $0.760-5.931$ & 0.151 \\
\hline Amplification & & & & & & & \\
\hline$>2$ regions & Yes/no & 8.771 & $2.673-28.571$ & $<0.001$ & 4.219 & $0.849-20.8333$ & 0.078 \\
\hline
\end{tabular}

OCCC, ovarian clear cell carcinoma; HR, hazard ratio; CI, confidence interval.

Table VI. Copy number alterations and chemosensitivity of the OCCC cases.

\begin{tabular}{|c|c|c|c|c|c|c|c|c|c|c|}
\hline \multirow[b]{2}{*}{ Amplification } & \multicolumn{2}{|c|}{$8 \mathrm{p} 11.22$} & \multicolumn{2}{|c|}{$8 \mathrm{p} 11.21$} & \multicolumn{2}{|c|}{$12 \mathrm{p} 13.31$} & \multicolumn{2}{|c|}{$20 \mathrm{q} 13.2$} & \multirow[b]{2}{*}{$>2$ regions } & \multirow[b]{2}{*}{$\leq 2$ regions } \\
\hline & Yes & No & Yes & No & Yes & No & Yes & No & & \\
\hline Sensitive & 15 & 46 & 6 & 55 & 3 & 58 & 8 & 53 & 1 & 60 \\
\hline Resistant & 8 & 18 & 4 & 22 & 6 & 20 & 5 & 21 & 4 & 22 \\
\hline Total & 23 & 64 & 10 & 77 & 9 & 78 & 13 & 74 & 5 & 82 \\
\hline \multirow[t]{2}{*}{ P-value } & \multicolumn{2}{|c|}{0.550} & \multicolumn{2}{|c|}{0.458} & \multicolumn{2}{|c|}{0.011} & \multicolumn{2}{|c|}{0.464} & \multicolumn{2}{|c|}{0.012} \\
\hline & \multicolumn{2}{|c|}{$3 q 26.1$} & \multicolumn{2}{|c|}{$4 q 13.2$} & \multicolumn{2}{|c|}{$22 \mathrm{q} 11.23$} & & & & \\
\hline Deletion & Yes & No & Yes & No & Yes & No & & & & \\
\hline Sensitive & 13 & 48 & 11 & 50 & 1 & 60 & & & & \\
\hline Resistant & 13 & 13 & 5 & 21 & 2 & 24 & & & & \\
\hline Total & 26 & 61 & 16 & 71 & 3 & 84 & & & & \\
\hline P-value & \multicolumn{2}{|c|}{0.008} & \multicolumn{2}{|c|}{0.895} & \multicolumn{2}{|c|}{0.157} & & & & \\
\hline
\end{tabular}

P-values were calculated by the Chi-square test. OCCC, ovarian clear cell carcinoma.

\section{Funding}

The present study was supported by Grants-in-Aid for Scientific Research on Innovative Areas (Integrative Analysis and Regulation of Cellular Diversity, no. 17H06325) from MEXT, Japan, and Project for Cancer Research and Therapeutic Evolution (P-CREATE, no. 17cm0106103h0002) from the Japan Agency for Medical Research and Development.

\section{Availability of data and materials}

The datasets used during the present study are available from the corresponding author upon reasonable request.

\section{Authors' contributions}

AM, TH, AO and TA conceived and designed the experiments. AM, MK, YK, TI and KS performed the experiments. AM, TH and TA analyzed the data. MU and AO provided reagents/materials/analysis tools. AM, TH and TA wrote the report. All authors read and approved the manuscript and agree to be accountable for all aspects of the research in ensuring that the accuracy or integrity of any part of the study are appropriately investigated and resolved.

\section{Ethics approval and consent to participate}

All the OCCC patients provided written informed consent. This study was performed in accordance with the Helsinki Declaration and was approved by the Ethics Committee of the Jikei University School of Medicine

\section{Consent for publication}

Not applicable.

\section{Competing interests}

The authors state that they have no competing interests. 


\section{References}

1. Gilks CB, Ionescu DN, Kalloger SE, Köbel M, Irving J, Clarke B, Santos J, Le N, Moravan V and Swenerton K; Cheryl Brown Ovarian Cancer Outcomes Unit of the British Columbia Cancer Agency: Tumor cell type can be reproducibly diagnosed and is of independent prognostic significance in patients with maximally debulked ovarian carcinoma. Hum Pathol 39: 1239-1251, 2008.

2. Matsuda T, Marugame T, Kamo K, Katanoda K, Ajiki W and Sobue T; Japan Cancer Surveillance Research Group: Cancer incidence and incidence rates in Japan in 2006: Based on data from 15 population-based cancer registries in the monitoring of cancer incidence in Japan (MCIJ) project. Jpn J Clin Oncol 42: 139-147, 2012.

3. Mackay HJ, Brady MF, Oza AM, Reuss A, Pujade-Lauraine E, Swart AM, Siddiqui N, Colombo N, Bookman MA, Pfisterer J, et al; Gynecologic Cancer InterGroup: Prognostic relevance of uncommon ovarian histology in women with stage III/IV epithelial ovarian cancer. Int J Gynecol Cancer 20: 945-952, 2010.

4. Duska LR, Garrett L, Henretta M, Ferriss JS, Lee L and Horowitz N: When 'never-events' occur despite adherence to clinical guidelines: The case of venous thromboembolism in clear cell cancer of the ovary compared with other epithelial histologic subtypes. Gynecol Oncol 116: 374-377, 2010.

5. Kuo KT, Mao TL, Jones S, Veras E, Ayhan A, Wang TL, Glas R, Slamon D, Velculescu VE, Kuman RJ, et al: Frequent activating mutations of PIK3CA in ovarian clear cell carcinoma. Am J Pathol 174: 1597-1601, 2009.

6. Kuo KT, Mao TL, Chen X, Feng Y, Nakayama K, Wang Y, Glas R, Ma MJ, Kurman RJ, Shih IeM, et al: DNA copy numbers profiles in affinity-purified ovarian clear cell carcinoma. Clin Cancer Res 16: 1997-2008, 2010.

7. Tan DS, Iravani M, McCluggage WG, Lambros MB, Milanezi F, Mackay A, Gourley C, Geyer FC, Vatcheva R, Millar J, et al: Genomic analysis reveals the molecular heterogeneity of ovarian clear cell carcinomas. Clin Cancer Res 17: 1521-1534, 2011.

8. Okamoto A, Sehouli J, Yanaihara N, Hirata Y, Braicu I, Kim BG, Takakura S, Saito M, Yanagida S, Takenaka M, et al: Somatic copy number alterations associated with Japanese or endometriosis in ovarian clear cell adenocarcinoma. PLoS One 10: e0116977, 2015.

9. Tian C, Kosoy R, Lee A, Ransom M, Belmont JW, Gregersen PK and Seldin MF: Analysis of East Asia genetic substructure using genome-wide SNP arrays. PLoS One 3: e3862, 2008.

10. Miotke L, Lau BT, Rumma RT and Ji HP: High sensitivity detection and quantitation of DNA copy number and single nucleotide variants with single color droplet digital PCR. Anal Chem 86: 2618-2624, 2014.
11. Langmead B and Salzberg SL: Fast gapped-read alignment with Bowtie 2. Nat Methods 9: 357-359, 2012.

12. Li H, Handsaker B, Wysoker A, Fennell T, Ruan J, Homer N, Marth G, Abecasis G and Durbin R; 1000 Genome Project Data Processing Subgroup: The sequence alignment/map format and SAMtools. Bioinformatics 25: 2078-2079, 2009.

13. Omura N, Mizuma M, MacGregor A, Hong SM, Ayars M, Almario JA, Borges M, Kanda M, Li A, Vincent A, et al: Overexpression of ankyrin1 promotes pancreatic cancer cell growth. Oncotarget 7: 34977-34987, 2016.

14. Guo JP, Shu SK, He L, Lee YC, Kruk PA, Grenman S, Nicosia SV, Mor G, Schell MJ, Coppola D, et al: Deregulation of IKBKE is associated with tumor progression, poor prognosis, and cisplatin resistance in ovarian cancer. Am J Pathol 175: 324-333, 2009.

15. Brocker CN, Vasiliou V and Nebert DW: Evolutionary divergence and functions of the ADAM and ADAMTS gene families. Hum Genomics 4: 43-55, 2009.

16. Marsit CJ,Zheng S, Aldape K, Hinds PW, Nelson HH, Wiencke JK and Kelsey KT: PTEN expression in non-small-cell lung cancer: Evaluating its relation to tumor characteristics, allelic loss, and epigenetic alteration. Hum Pathol 36: 768-776, 2005.

17. Taniue K, Kurimoto A, Sugimasa H, Nasu E, Takeda Y, Iwasaki K, Nagashima T, Okada-Hatakeyama M, Oyama M, Kozuka-Hata $\mathrm{H}$, et al: Long noncoding RNA UPAT promotes colon tumorigenesis by inhibiting degradation of UHRF1. Proc Natl Acad Sci USA 113: 1273-1278, 2016.

18. Barrow J, Adamowicz-Brice M, Cartmill M, MacArthur D, Lowe J, Robson K, Brundler MA, Walker DA, Coyle B and Grundy R: Homozygous loss of ADAM3A revealed by genomewide analysis of pediatric high-grade glioma and diffuse intrinsic pontine gliomas. Neuro-oncol 13: 212-222, 2011.

19. Asnaghi L, Alkatan H, Mahale A, Othman M, Alwadani S, Al-Hussain H, Jastaneiah S, Yu W, Maktabi A, Edward DP, et al: Identification of multiple DNA copy number alterations including frequent 8p11.22 amplification in conjunctival squamous cell carcinoma. Invest Ophthalmol Vis Sci 55: 8604-8613, 2014.

20. Dekker J: Gene regulation in the third dimension. Science 319: 1793-1794, 2008.

21. Andrey G, Montavon T, Mascrez B, Gonzalez F, Noordermeer D, Leleu M, Trono D, Spitz F and Duboule D: A switch between topological domains underlies HoxD genes collinearity in mouse limbs. Science 340: 1234167, 2013.

22. Nora EP, Lajoie BR, Schulz EG, Giorgetti L, Okamoto I, Servant N, Piolot T, van Berkum NL, Meisig J, Sedat J, et al: Spatial partitioning of the regulatory landscape of the X-inactivation centre. Nature 485: 381-385, 2012. 